\title{
The long bar as seen by the VVV Survey
}

\author{
II. Star counts $\star$
}

E. B. Amôres ${ }^{1,2,3}$, M. López-Corredoira ${ }^{4,5}$, C. González-Fernández ${ }^{6}$, A. Moitinho ${ }^{3}$, D. Minniti ${ }^{7,8,9}$, and S. Gurovich ${ }^{10,11}$

${ }^{1}$ UEFS, Departamento de Física, Av. Transnordestina, S/N, Novo Horizonte, CEP 44036-900, Feira de Santana, Brazil e-mail: ebamores@uefs.br

${ }^{2}$ UEFS, Observatório Astronômico Antares, Rua da Barra, 925, Jardim Cruzeiro, CEP 44024-432, Feira de Santana, Brazil

3 SIM, Faculdade de Ciências da Universidade de Lisboa, Ed. C8. Campo Grande, 1749-016 Lisbon, Portugal

${ }^{4}$ IAC, vía Láctea s/n, 38200 La Laguna (Tenerife), Spain

5 Departamento de Astrofísica, Universidad de La Laguna, 38206 La Laguna, Tenerife, Spain

6 Departamento de Física, Ingeniería de Sistemas y Teoría de la Señal, Universidad de Alicante, Apdo. 99, 03080 Alicante, Spain

7 Departamento Astronomía y Astrofísica, Pontificia Universidad Católica de Chile, Av. Vicuña Mackenna 4860, Santiago, Chile

8 Vatican Observatory, 00120 Vatican City State, Italy

9 Departamento de Ciencias Físicas, Universidad Andres Bello, 8370134 Santiago, Chile

10 Observatorio Astronómico de Córdoba, Universidad Nacional de Córdoba, Laprida 854, 5000 Córdoba, Argentina

11 Instituto de Astronomía Teórica y Experimental (IATE-CONICET), Laprida 922 X5000BGR Córdoba, Argentina

Received 20 June 2012 / Accepted 20 June 2013

\section{ABSTRACT}

\begin{abstract}
Context. There is still some debate about the presence and the morphological properties of the long bar in the inner Galaxy.
Aims. We investigate the morphological properties of the long Galactic bar using the VVV survey extending star counts at least 3 mag deeper than 2MASS. Our study covers the relatively unexplored negative longitudes of the Galactic bar. We obtain a detailed description of the spatial distribution of star counts towards the long Galactic bar as well as to measure its parameters.

Methods. We performed star counts towards $-20^{\circ}<\ell<0,|b| \leq 2^{\circ}$ using VVV, 2MASS, and GLIMPSE data. We applied an average interstellar extinction correction. We also adjusted latitudinal profiles to obtain the centroid variation and bar thickness.

Results. We probe the structure of long Galactic bar, as well as its far edge at $\ell \approx-14^{\circ}$. The differences between counts with and without extinction correction allow us to produce a crude extinction map showing regions with high extinction, mainly beyond the end of long Galactic bar. The latitudinal profiles show evidence of the centroid vertical variation with Galactic longitude reaching a minimum at $\ell \approx-13.8^{\circ}$. The bar has an inclination angle $\alpha=43^{\circ} \pm 5^{\circ}$ with respect to the line Sun-Galactic center. In addition, we have determined the bar parameters, such as thickness, length, and stellar distribution.
\end{abstract}

Key words. ISM: structure - dust, extinction - Galaxy: stellar content - Galaxy: structure - Galaxy: center - Galaxy: general

\section{Introduction}

The understanding of the structure that can be attributed to a bar in the Galactic center has significantly advanced in the past three decades, mainly thanks to the IRAS, COBE/DIRBE, TMGS, DENIS, 2MASS, and UKIDSS infrared surveys. These surveys allowed us to increase the knowledge obtained from the Hayakawa et al. (1981) and Matsumoto et al. (1982) maps, not only of the structure, but also of the shape of the Galactic center (Blitz \& Spergel 1991, hereafter BL91).

After studying the asymmetries in the Galactic center, Lizst \& Burton (1980) and Burton \& Lizst (1983) suggested they could be attributed to a bar with an inclination angle of approximately $25^{\circ}$. BL91 argued for the existence of asymmetries in the photometric images of the Galactic bulge by analyzing the $2.4 \mu \mathrm{m}$ observations performed by Matsumoto et al. (1982) and of Weiland et al. (1994) based on the images obtained by the COBE/DIRBE experiment in $1.25,2.2,3.5$, and $4.9 \mu \mathrm{m}$. The asymmetry is also visible in star counts (e.g., Stanek et al. 1994; Hammersley et al. 1994), which show systematically more stars at positive Galactic longitudes (within $<30^{\circ}$ ) and close to the

* Appendix $\mathrm{A}$ is available in electronic form at http://www . aanda.org
Galactic plane, compared to negative longitudes. It was also supported by the distribution of red clump stars (Hammersley et al. 2000; Cabrera-Lavers et al. 2007a), the dynamics of the stellar and the gaseous components in the Galactic center (e.g., Minchev et al. 2007), gravitational micro-lensing toward the Galactic bulge (Paczynski et al. 1994; Popowski et al. 2005), and the kinematic effects on the local disk stars (Gardner \& Flynn 2010; Romero-Gómez et al. 2011).

While some papers refer to a thick triaxial structure (bulge or thick bar) with a semi-major axis length of $2.5 \mathrm{kpc}$ and a position angle (PA) of $15^{\circ}-30^{\circ}$ with respect to the Sun-Galactic center direction (e.g., López-Corredoira et al. 2005; Habing et al. 2006; Rattenbury et al. 2007; Vanhollebeke et al. 2009; Robin et al. 2012), other contributions suggest that there is also a long thin bar, the in-plane bar, with a half length of $4 \mathrm{kpc}$ and a position angle of around $45^{\circ}$ : e.g., López-Corredoira et al. (2007, LC07 and references therein), Cabrera-Lavers et al. (2007a), Vallenari et al. (2008), Churchwell et al. (2009).

This long bar has a tip in the positive longitude at the beginning of the Scutum's arm (Dame et al. 1986). As realized by Sevenster et al. (1999), the angle is around $25^{\circ}$ or lower when low latitudes are excluded from the fit, and around $40-45^{\circ}$ in the plane regions within $-15^{\circ}<\ell<30^{\circ}$. Furthermore, there 
is some research on a possible third component, a nuclear bar (Alard 2001; Nishiyama et al. 2005; González et al. 2011) within $|\ell|<4^{\circ}$ in the plane. Some of us think that this is a more uncertain structure, since it is very dependent on a correct subtraction of the bulge+long bar, the excess of very bright stars due to star forming regions in the inner bulge (López-Corredoira et al. $2001 \mathrm{~b}$ ), or the errors affecting the distance of the red clump stars (see Appendix in LC01).

Rather than two large structures, it was suggested by Martínez-Valpuesta \& Gerhard (2011) a scenario of a single structure with a twisted major axis. In principle, we find this proposal acceptable from a purely morphological point of view. In any case, the concepts are different: bars or pseudo-bulges form as a result of instability in differentially rotating disks (Sellwood 1981), whereas bulges are primordial Galactic components. Therefore, the scenario bulge+bar is not the same thing as a single structure, and one should observe other differences apart from the morphology. We think that the particular features of this new proposal of a single twisted bulge/bar scenario leaves certain observational facts unexplained, such as the star formation regions at the tips of the long bar, whereas the model of a misaligned bulge + long bar successfully explains them (López-Corredoira et al. 2011).

In addition, there was evidence of kinematic differences between metal-rich and metal-poor populations in the bulge (Minniti et al. 1996). We now know that there are two distinct populations in the thick bulge: a metal-rich population with barlike kinematics and a metal-poor population with kinematics corresponding to an old spheroid or a thick disk, so the two main scenarios for the bulge formation co-exist within the Milky Way bulge (Babusiaux et al. 2010).

The largest (deepest) near-infrared survey of the Milky Way (MW) disk and bulge is the VISTA Variables in the Via Láctea, hereafter VVV (Minniti et al. 2010; Saito et al. 2012), an important observational base for studying the Galactic structure. Works with VVV data, such as those of Saito et al. (2011) and González et al. (2011, 2012), have already provided new insight in to the inner structure of our Galaxy.

Here we use the combined 2MASS, VVV, and GLIMPSE products to explore the long bar at negative longitudes $\left(-20^{\circ}<\right.$ $\ell<0,|b| \leq 2^{\circ}$ ), which has been less explored than the positive longitude counterpart. In particular, star counts are performed in the $K_{\mathrm{s}}$ band for investigating the shape and structure of the bar at negative longitudes and computing its parameters (axis dimension, edges, size, inclination angle, etc.). Our study follows the approach of López-Corredoira et al. (2001a, hereafter LC01), who performed a detailed analysis of star counts in the long bar region $\left(|\ell| \leq 30^{\circ},|b| \leq 2^{\circ}\right)$ based on TMGS and DENIS data. A comparison with the different extinction methods used for general extinction corrections (Majewski et al. 2011 and LC01) is also presented.

This is the second in a series of two papers addressing the Galactic bar as seen by the VVV survey. Here, we focus on results from star counts. The first paper is dedicated to the analysis of color-magnitude diagrams (González-Fernández et al. 2012, hereafter Paper I).

The remainder of this paper is organized as follows. Section 2 presents the VVV, 2MASS, and GLIMPSE data used in this work, including calibration, source matching in both surveys, elimination of duplicated stars in the VVV tiles, and elaboration of the VVV catalogs. Section 3 presents the method for correcting the effects of interstellar extinction in 2MASS, VVV, and GLIMPSE data and for defining their completeness limits. An analysis of the latitudinal and longitudinal ${ }^{1}$ star counts profiles is presented in Sect. 4. Section 5 is dedicated to the resulting bar maps, parameters, and their discussion. Finally, Sect. 6 addresses the conclusions of this study and gives some final remarks.

\section{Data}

\subsection{VISTA variables in the via Láctea (VVV)}

VVV is an ESO public variability survey with the 4-m VISTA telescope at Cerro Paranal (Minniti et al. 2010). It performs observations in the $Z, Y, J, H$, and $K_{\mathrm{s}}$ near infrared (NIR) bands towards the Galactic bulge and part of the disk, covering a total area of 562 square degrees. VVV is in its fourth year. Full disk and bulge coverage in $Z, Y, J, H$, and $K_{\mathrm{s}}$ bands has been secured. For a detailed description of the observations during the first year of the survey, see Saito et al. (2012). The data were reduced using the CASU pipeline ${ }^{2}$.

This work makes use of 56 tiles covering a region of approximately 90 square degrees. The tile identification labels and their coordinates are presented in Saito et al. (2012, Table A.1). It is well established that long bar ends near $\ell \sim-15^{\circ}$ (LC01 and references therein). For this reason, our analysis is restricted to Galactic longitudes within $\ell \sim-20.0^{\circ}$ to $0.0^{\circ}$.

We take the VVV tile catalogs toward the bar (including disk and bulge) in each individual $J, H$, and $K_{\mathrm{s}}$ filters (version 1.1) to create multi band merged catalogs for each tile region. A bandmerging python code was developed that emulates the method being implemented by VISTA Science Archive with search radius equal to 1.0 arcsec. For details on the VVV and 2MASS (Skrutskie et al. 2006) cross-match, see Paper I.

The sources used in this work are all gathered in a single file. Only sources classified as stellar (VVV-icls classification flag equal to -1 ) by VVV pipeline in both $J$ and $K_{\mathrm{s}}$ filters were used. The file includes the source and tile identifiers, magnitude, magnitude error, and a flag to indicate whether the source was taken from 2MASS or from VVV.

The VVV and 2MASS photometry of stars in common were compared and the difference in magnitude for each star in each filter was calculated. For most tiles, the average differences in $J$ and $K_{\mathrm{s}}$ were less than $0.1 \mathrm{mag}$; however, for eight tiles (b327, b328, b329, b330, b331, b332, d070 and d071), we noticed differences in $K_{\mathrm{s}}$ ranging from 0.2 to $0.7 \mathrm{mag}$, both positive and negative. For these tiles we opted to transform the VVV photometry to the 2MASS system by adding the corresponding photometric offsets.

The VVV observational strategy was designed to have observations of the same sources in a small overlap of contiguous tiles, which is useful not only for variability studies but also for calibration purposes. In this way, tiles contain overlap regions that correspond to $6 \%$ of the observed sources for a given tile. As we work with star counts, it is necessary to identify these sources in order to avoid artifacts due to duplication of counts.

Duplicate stars were eliminated as follows: i) for each tile we computed its limits (lower and higher) for both Galactic longitude and latitude; ii) from these limits we verified, for each tile, the possible same sources considering a match of 1 arcsec within a strip delimited by 0.3 degrees (chosen in order to have a generous search region for the detection of duplicate stars) for

\footnotetext{
1 Latitudinal profiles are the distribution of star counts vs. latitude and longitudinal profiles are the distribution of star counts vs. longitude.

2 http://casu.ast.cam.ac.uk/
} 

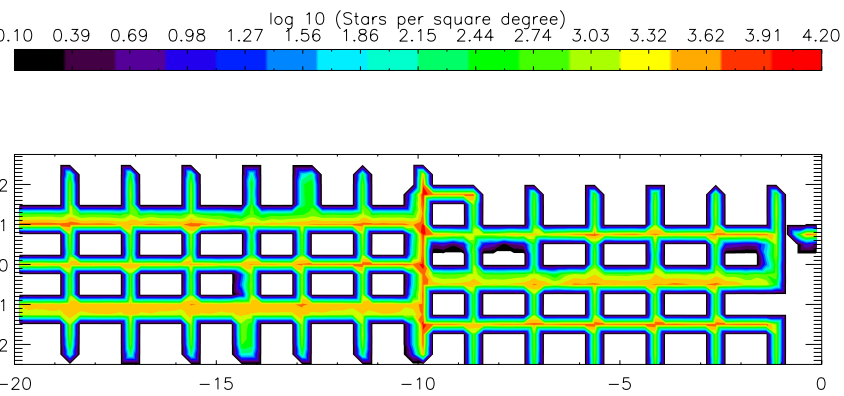

Fig. 1. Tiles with overlap regions. Star counts binned in $(\Delta l=\Delta b=$ $0.25^{\circ}$ ). Colors represent the number of duplicated stars in log. White means no duplicated stellar counts in that area.

each side of a rectangle for all other tiles; iii) since one source in other tile is identified as similar source of the reference tile, we quoted it as a flag pointing to a duplicated source. Applying this analysis we have found 1663426 sources that were eliminated from our study leaving 27687811 stars with valid photometry for both $J$ and $K_{\mathrm{s}}$.

Figure 1 shows a map with counts for the overlapping regions. One can identify that typical counts in the densest regions range from 3000 to 7000 stars. A tilt feature can be seen at different latitude ranges toward $\ell \sim-10^{\circ}$ that can be attributed to the fact that tile centers are located at different latitudes when considering $\ell<-10^{\circ}$ and $\ell>-10^{\circ}$, respectively. White regions mean no duplicated counts. The counts are irregular, and eliminating duplicated sources is mandatory for star counts studies toward any large VVV region.

\subsection{GLIMPSE}

The GLIMPSE data (Benjamin et al. 2003; Churchwell et al. 2009) for all phases of the project (GLIMPSE I, II and 3D) are available at IPAC webpage ${ }^{3}$. As each phase corresponds to specific regions with different spatial coverage in both longitude and latitude towards the Galactic plane, we have selected all of them toward $-20^{\circ}<\ell<0^{\circ}$. Once the overlaping regions were identified, we followed the GLIMPSE team recommendation (see footnote 3 ) to use the most recent data, as for instance, GLIMPSE II data for the region that extends from $\ell \sim-11.0^{\circ}$ to $-10.0^{\circ}$ even though data for this region is available in both GLIMPSE I and II.

Care was taken to limit the overlap regions to avoid duplicate sources in the star counts analysis. For each phase of the project there are separated files that correspond to a specific range of Galactic longitude, one degree wide. We have joined these files in a single file for each phase with: Galactic coordinates, magnitudes, and the errors in GLIMPSE filters: [3.6], [4.5], [5.8], and [8.0] $\mu \mathrm{m}$, as well as 2MASS photometry (magnitudes and errors) in $J, H$, and $K_{\mathrm{s}}$.

Interstellar extinction is lower in the mid-IR (MIR) bands (e.g. [8.0] $\mu \mathrm{m}$ ) than in optical or NIR. Stellar spectral energy distributions sampled in the MIR have similar shapes, independent of stellar type. This allows a more precise determination of extinction through the use of adequate color combinations. Based on these ideas, Majewski et al. (2011, hereafter M11) developed a method termed Rayleigh-Jeans color excess (RJCE) based on 2MASS and GLIMPSE data providing useful relations to determine interstellar extinction, see Eq. (1) of M11. Further

\footnotetext{
3 http://irsa.ipac.caltech.edu/data/SPITZER/GLIMPSE
}

details on extinction corrections in GLIMPSE data are given in Sect. 3.2.

Since VVV is deeper than 2MASS, we choose to increase our matched source catalog by also matching GLIMPSE data against our VVV source data (combined $J, H$ and $K_{\mathrm{s}}$ photometry).

Because the extinction correction method of M11 also requires the $H$ magnitude, we have compiled another matched VVV catalog of stellar sources with reliable photometry in $J$, or $H$, or $K_{\mathrm{s}}$ and applied the same procedure as described in the previous section for identifying duplicate sources in the tiles. This resulted in a catalog of positions and photometry for 55793434 VVV sources (after discarding 6\% of the duplicates).

The difference between the number of sources in this second catalog in relation to the first one mentioned in Sect. 2.1 is the result of it containing all sources with at least one detection in one filter, contrary to the first one that needs to have detection for both $J$ and $K_{\mathrm{s}}$. It should be noted that to compute GLIMPSE counts at $[8.0] \mu \mathrm{m}$ as indicated by Eq. (5), it is mandatory that a source be detected in [4.5], [8.0] $\mu \mathrm{m}$, and $H$ filters. In summary, for the analysis of the VVV+2MASS (Sect. 3.1) we only use $J$ and $K_{\mathrm{s}}$. However, in this second catalog only $3 \%$ of the sources are detected for both $J$ and $K_{\mathrm{s}}$ and not at $H$.

Next, we cross-matched this catalog (by selecting the nearest source within a search radius equal to $1^{\prime \prime}$ ) with the GLIMPSE merged catalog mentioned above producing another catalog with coordinates and VVV and GLIMPSE magnitudes (and errors). Table 1 summarizes GLIMPSE limits for both Galactic longitude and latitude for each phase of the project used in the present work, as well as the comparison of the number of sources obtained with common photometry $J, H$, and $K_{\mathrm{s}}$ using only 2MASS data as provided by GLIMPSE merged catalog and VVV+2MASS catalog produced by us. The number of matches increases significantly using VVV photometry instead of only GLIMPSE+2MASS.

As can be seen in Table 1, by adding the matched GLIMPSE+VVV+2MASS source catalog to our analysis, a substantial increase (a factor $>4$ for GLIMPSE II) is obtained in matched source numbers, as compared to a 2MASS (only) cross matches. Since use of VVV data has the advantage of getting to a larger number of fainter and redder sources, we can expect that the extinction correction will be significantly more precise.

\section{Extinction and completeness limits}

Interstellar extinction plays an important role in analysis in the Galactic plane, as shown in Amôres \& Lépine (2005, hereafter AL05) and Robin (2009, and references therein). Assessment of extinction is essential for a correct star counts analysis. Without it, analysis can become severely biased, especially in high extinction areas.

Below, we outline the methods adopted in correcting for extinction and defining completeness limits for the VVV+2MASS and GLIMPSE data sets. A detailed analysis of the interstellar extinction toward VVV fields is beyond the scope of this present paper, and it is presented in others papers of the VVV collaboration: e.g., González et al. (2012) and Chen et al. (2013).

\subsection{VVV}

We follow the method described in LC01 for determining an extinction free magnitude. It should be noted that this method is also quite similar to the one presented by Alard (2002). As 
A\&A 559, A11 (2013)

Table 1. List of the coverage for GLIMPSE data used in the present work.

\begin{tabular}{lccccc}
\hline \hline Data release & Gal. longitude & Gal. latitude & 2MASS & 2MASS+VVV & Only GLIMPSE \\
\hline GLIMPSE I & $-20^{\circ} \leq \ell \leq-11^{\circ}$ & $|b| \leq 1^{\circ}$ & 998451 & 3253321 & 4174079 \\
GLIMPSE II & $-11^{\circ} \leq \ell \leq 0^{\circ}$ & $|b| \leq 1^{\circ}$ & 1826965 & 8320253 & 11077393 \\
GLIMPSE III & $-20^{\circ} \leq \ell \leq 0^{\circ}$ & $|b| \leq 2^{\circ}$ & 1347374 & 2999419 & 4066203 \\
\hline
\end{tabular}

Notes. The columns $2 M A S S$ and $2 M A S S+V V V$ are respectively the number of valid sources detected in the three filters $\left(J, H\right.$ and $\left.K_{\mathrm{s}}\right) ;$ only GLIMPSE means sources detected only by GLIMPSE. For GLIMPSE II, the latitudes limits expand to $b \pm 1.5$ and \pm 2.0 for longitude ranges $-5^{\circ} \leq \ell \leq-2^{\circ}$ and $-2^{\circ} \leq \ell \leq 0^{\circ}$, respectively.

pointed out by LC01, the method used in the present paper is roughly consistent with that of Schultheis et al. (1999), except that here each star is individually corrected for extinction before each area is averaged. Anyway, the method is also validate considering star counts simulations as presented in Appendix A.

Since the sources with $J-K_{\mathrm{s}}<0.5$ are dominated by local disk dwarfs (see Robin et al. 2003 and Marshall et al. 2006), we have not used them in our star counts method. Of course, there will also be disk stars with $J-K_{\mathrm{s}}>0.5$. However, as pointed by LC01, this will be a small proportion of the total sources and their distribution is symmetrical and in principle predictable, so at most there will be a loss of contrast for the inner Galaxy features. For the case where the majority of the sources are relatively concentrated in a certain location along the line of sight, this is therefore a straightforward method for recovering the form of the underlying star distribution, at least in the infrared.

Moreover, this approach is reasonable (LC01) thanks to the high density of sources in the inner Galaxy. In many regions, over $50 \%$ of the sources at a particular magnitude are from the inner Galaxy, so the vast majority of the detected sources come from a relatively restricted distance range. The correction holds for stars satisfying $J-K_{\mathrm{s}} \geq\left(J-K_{\mathrm{s}}\right)_{0}$ and is given by

$m_{K_{\mathrm{s}}, \text { correc. }}=m_{K_{\mathrm{s}}}-\frac{A_{K_{\mathrm{s}}}}{A_{J}-A_{K_{\mathrm{s}}}} \operatorname{Max}\left[0,\left(J-K_{\mathrm{s}}\right)-\left(J-K_{\mathrm{s}}\right)_{0}\right]$,

where

$\frac{A_{K_{\mathrm{s}}}}{A_{J}-A_{K_{\mathrm{s}}}}=\frac{3}{5}$

Since some stars will have color bluer than the average of the whole population, these stars should have non physical negative extinction. To avoid applying non physical correction, their extinction values are set to 0 , and therefore for stars with $J-K_{\mathrm{s}}<$ $\left(J-K_{\mathrm{s}}\right)_{0}$, the corrected magnitude equals the observed one: $m_{K_{\mathrm{s}}, \text { correc. }}=m_{K_{\mathrm{s}}}$ in Eq. (1).

The value of 0.6 in Eq. (1) was obtained considering an average value from Rieke \& Lebofsky (1985) and Glass (1999) that obtained 0.64 and 0.55 , respectively, for the ratio $\frac{A_{K_{\mathrm{s}}}}{A_{J}-A_{K_{\mathrm{S}}}}$. We have considered $\left(A_{V}: A_{J}: A_{K_{\mathrm{s}}}=1.0: 0.282: 0.112\right)$ and $\left(A_{V}: A_{J}: A_{K_{\mathrm{s}}}=1.0: 0.256: 0.089\right)$ for the Rieke \& Lebofsky (1985) and Glass (1999) extinction coefficients, respectively.

That we have used a single $\left(J-K_{\mathrm{s}}\right)_{0}$, even considering there is a transition between the population content of the bar/bulge and the disk, could be justified by the very slight modification of the average color. Star formation regions are more abundant in the disk that has younger populations and an excess of supergiants and bright giants, but these very bright stars are still much scarcer than other fainter stars.
The red clumps have more or less the same intrinsic color ${ }^{4}$, and will have different reddening from the disk and from the bulge, but the counts of red clumps are dominated by the bulge/bar. The simulations in our Appendix A illustrate this.

To verify that adopting $\left(J-K_{\mathrm{s}}\right)_{0}=1.0$ is a valid value for the intrinsic color $\left(J-K_{\mathrm{s}}\right)_{0}$ in Eq. (1), we have generated a series of sets of corrected $K_{\mathrm{s}}$ photometry using $\left(J-K_{\mathrm{s}}\right)_{0}$ ranging from 0.6 to 1.6 at intervals of $0.2 \mathrm{mag}$. Each set is split in regions of $0.25^{\circ} \times 0.25^{\circ}$ square degrees. The area of $0.25^{\circ} \times 0.25^{\circ}$ square degrees for each region was used as a compromise between having a good spatial resolution (the lower the better) and having enough counts (the more the better). Moreover, using fields with this area reduces the effects for both interstellar extinction and crowding that changes significantly from neighboring regions in the Galactic plane. Star counts with 0.5 mag steps were then performed for each of these split regions. The appropriate value for the constant should be the one producing the lowest variation in the counts between neighboring regions.

Field crowding, interstellar extinction and variable conditions during the observations have an impact on star counts, so care must be taken to assure that all regions in our analysis are within their completeness limits. We therefore performed the analysis considering different magnitude ranges by imposing a series of magnitude cut-offs ranging from 9.0 to 14.0 (0.5 mag steps). Then, for each of these cut-offs and for each region, we computed the squared difference between the counts obtained for this direction and its eight neighboring regions, as

$\sigma_{i, j}^{2}=\frac{1}{8} \sum_{k, l}^{8} \frac{\left(N_{K, l}-N_{i, j}\right)^{2}}{N_{i, j}^{2}}$

where $N(K, l)$ is the number of stars for a given bin, and $N_{i, j}$ is each neighbor cell in $(\ell, b)$ space.

The final total measure of variation (for each magnitude cutoff) is defined as the square root of the sums of the square differences, divided by the number of regions $(n)$ :

$\sigma^{2}=\frac{1}{n} \sum_{i, j}^{n} \sigma_{i, j}^{2}$.

Figure 2 shows the measure of variation ( $\sigma$, from now on) as a function of the cut-off magnitude for the different trial values of the correction constant. The value of $\left(J-K_{\mathrm{s}}\right)_{0}=1.0$ clearly produces the lowest $\sigma$ up to the corrected $K_{\mathrm{s}} \sim 13.5 \mathrm{mag}$. It keeps the minimum for the range $12<K_{\mathrm{s}}<14$. The other value $\left(J-K_{\mathrm{s}}\right)_{0}=1.2$ is a little bit lower for $12<K_{\mathrm{S}}<13$, but it increases significantly for $13<K_{\mathrm{s}}<14$.

The estimate of the completeness limit for each field was performed using star counts and histograms with bin size equal

4 As pointed out by Sarajedini et al. (1995), the luminosity of red clumps has a small dependence for both age and metallicity. 
E. B. Amôres et al.: The long bar as seen by the VVV Survey. II.

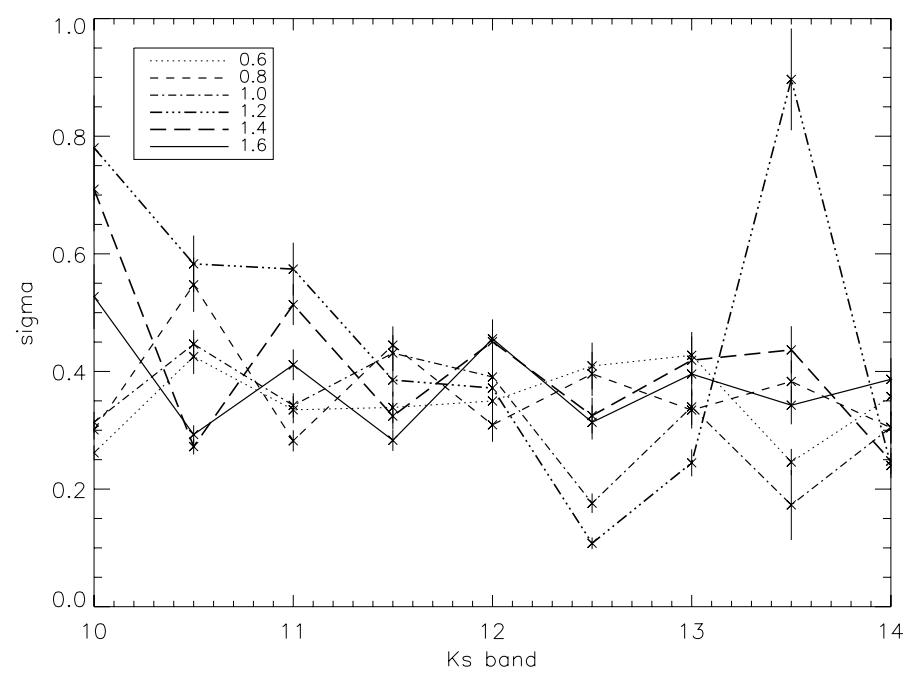

Fig. 2. $\sigma$ as computed in Eq. (2) as a function of the completeness limit for each value $\left(J-K_{\mathrm{s}}\right)_{0}$ as presented in the legend for all fields (with area equal to 0.0625 square degrees) used in the present work.

to 0.5 as a function of magnitude (also referred to as luminosity function). The peak of each histogram for each field was defined as the completeness limit. Later, we elaborated a map of the completeness limit as a function of Galactic latitude and longitude, which shown that after extinction correction (Eq. (1)) most of them, e.g., $95 \%$ of fields have $K_{\mathrm{s}} \geq 13.5$ mag as completeness limit. The fields that have completeness limits lower than $13.5 \mathrm{mag}$ are mainly located at $|\ell| \leq 2^{\circ}$. Since this region is not the focus of our work, we preferred to lose completeness for those few fields in order to have deep completeness along the entire bar.

We also analyzed the counts as a function of magnitude, and the value of $K_{\mathrm{s}} \sim 13.5 \mathrm{mag}$ represents that the stellar counts stop growing. In addition, choosing this value as the completeness limit also provides the best contrast in the star counts maps of the long bar. Similar analysis was performed in other NIR filters. The completeness for $J$ and $H$ filters were 17.0 and $16.0 \mathrm{mag}$, respectively.

\subsection{GLIMPSE}

As pointed out by M11, the use of NIR and MIR photometry has several advantages since extinction is noticeably lower than in the visible, and higher precision foreground extinction values can be determined when combining MIR with NIR photometry. We performed star counts using the combined VVV+GLIMPSE+2MASS data described in Sect. 2.2. As in the previous section, counts were performed considering grids of $0.25^{\circ} \times 0.25^{\circ}$ square degrees. Here, extinction is computed with Eq. (4). The completeness limits at [8.0] and [4.5] $\mu \mathrm{m}$ were determined for each field (as explained above), which are 11.0 and 10.5 mag in most cases. Using the relations provided by Rieke $\&$ Lebofsky (1985), the extinction at [8.0] $\mu \mathrm{m}$ is given by

$A([8.0 \mu \mathrm{m}])=0.020\left(A_{K_{\mathrm{s}}} / 0.112\right)$,

where $A_{K_{\mathrm{s}}}=0.112 A_{V}$.

To better correct the effects of interstellar extinction, we used Eq. (1) provided by M11:

$A\left(K_{\mathrm{s}}\right)=0.918(H-[4.5 \mu \mathrm{m}]-0.08)$.

The result of the combined VVV+2MASS+GLIMPSE data dereddened using Eq. (4) is presented in Sect. 5.

\section{Star counts}

The following sections analyze the star counts from two perspectives: their longitudinal and latitudinal profiles.

\subsection{Longitudinal counts}

One effective way to study counts is to analyze their variation with longitude (e.g., longitudinal profiles) for different latitude ranges. In this case we considered three different ranges: i) $|b| \leq 0.375^{\circ}\left(b=0^{\circ}, \pm 0.25^{\circ}\right)$; ii) $0.375^{\circ}<|b| \leq 1.125^{\circ}$ $\left(b= \pm 1.0,0.75,0.50^{\circ}\right)$; iii) $1.375^{\circ} \leq|b| \leq 2.125^{\circ}(b=$ $\pm 1.5,1.75,2.0^{\circ}$ ). Figure 3 (upper left panel) shows a longitudinal profile for the observed counts (corrected by interstellar extinction) up to $K_{\mathrm{s}}=13.5 \mathrm{mag}$ for the three latitude ranges mentioned above. The longitude bin width is $1^{\circ}$. A clear decrease in the counts until $\ell \sim-14^{\circ}$ for the three latitude ranges can be seen, most notably for Galactic latitudes located toward $|b| \leq 0.375^{\circ}$.

Another visible feature is the end of the bulge loss revealed by the change of slope in the decrease in star counts at $\ell \sim-8^{\circ}$ for the higher latitudes. For instance for $-8.0^{\circ}<\ell \leq-3.0^{\circ}$, the counts have an average value equal to $\sim 1.5 \times 10^{5}$ to $1.1 \times 10^{5}$ for $\ell \sim-8^{\circ}$. Also, it can be seen that counts for the mid latitude $\left(0.375^{\circ}<|b| \leq 1.125^{\circ}\right)$ are greater than for the low latitude $\left(|b| \leq 0.375^{\circ}\right)$ for some longitude ranges $\left(-5.0^{\circ}<\ell<-2.5^{\circ}\right.$ and $\ell>-16.5^{\circ}$ ). This latter feature can be accounted for by errors in the extinction correction and the shift in the stellar distribution centroid (see Sect. 4.2).

A rough visualization of the interstellar extinction toward the Galactic bar and bulge can be obtained by displaying the ratio between the counts with and without extinction correction. Figure 3 (upper right panel) depicts the variation in relative counts with longitude, which also provides a rough estimate of the variation in interstellar extinction. To produce this estimate, we have considered the relative difference between the corrected and uncorrected counts for each interval of one degree in longitude. The interval of Galactic latitude is shown in the legend of figures.

To compare the interstellar extinction values obtained in the present work with the obtained ones by other works, we have considered three different estimates covering two ranges of Galactic longitude: i) the map produced by González et al. (2012) using new and deep VVV data and red clump method covering $\left(-10^{\circ}<\ell<0^{\circ}\right)$; ii) the 3D interstellar extinction model proposed by Marshall et al. (2006) using both the Besançon Galaxy Model (Robin et al. 2003) and 2MASS data. In this last model, we used as extinction the value that corresponds to the maximum value of distance, ranging from 10 to $13 \mathrm{kpc}$, for Galactic longitudes covering the regions located at $-20^{\circ}<\ell<-10^{\circ}$; iii) the maps provided by Nidever et al. (2012) based on the Rayleigh-Jeans color excess method using GLIMPSE-I,-II,-3D data.

The maps of González et al. (2012) and Nidever et al. (2012) were available during the referee process, as well as the $3 \mathrm{D}$ extinction model provided by Chen et al. (2013). We have not used this last one in our comparison since, as pointed out by Chen et al. (2013), the results for the region of present study are in good agreement with ones obtained by González et al. (2012).

It should be noted that each point of extinction obtained by the method provided by other authors was obtained considering the median of extinction for an interval of one degree in Galactic longitude. For comparison, Fig. 3 (lower left panel) displays the average interstellar extinction distribution provided by 

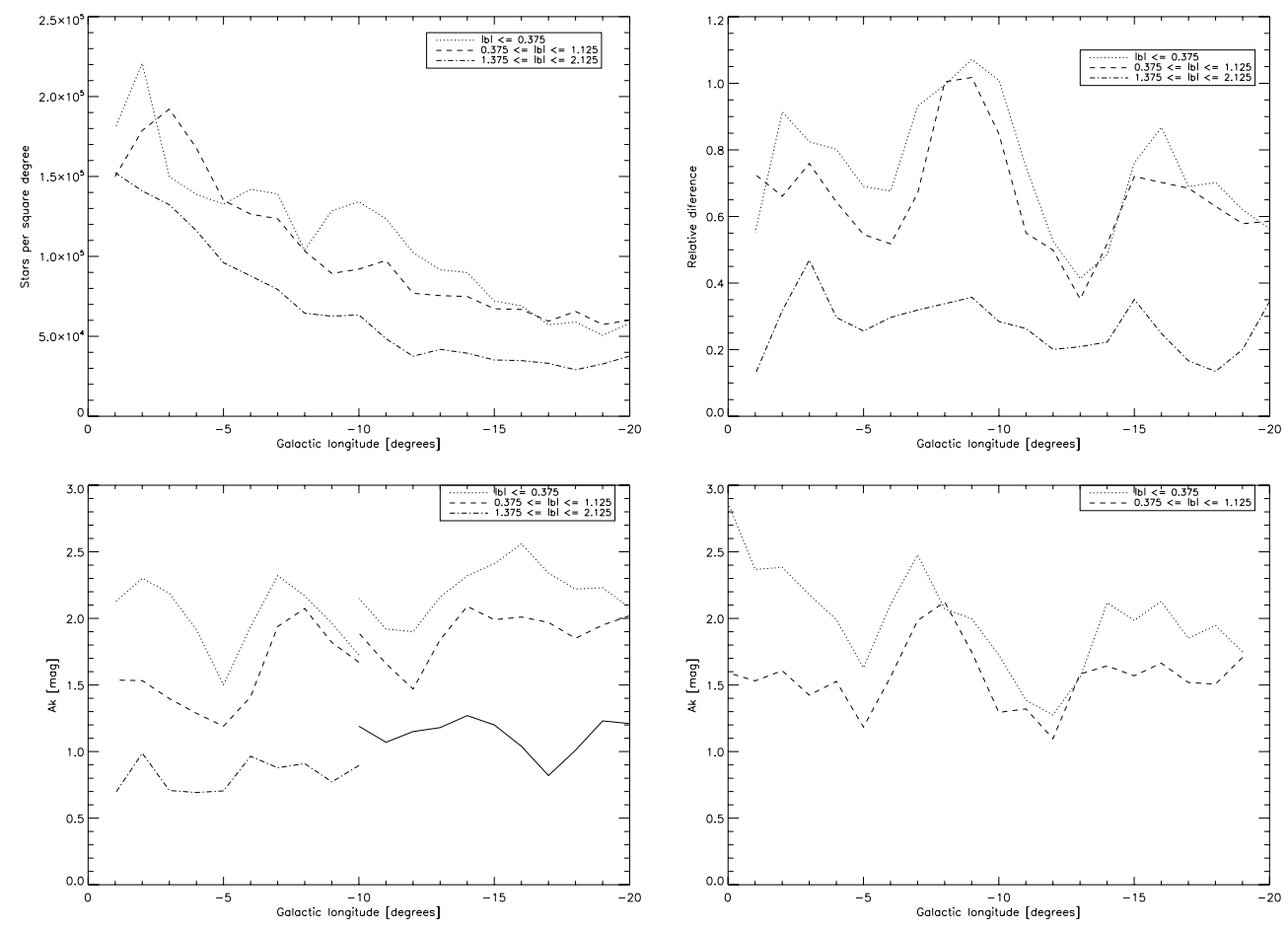

Fig. 3. Longitudinal profile for three ranges of latitude as mentioned in the legend. Upper left panel: star counts (for the observed counts up to $K_{\mathrm{s}}=13.5 \mathrm{mag}$ ) for magnitude corrected by extinction effects; upper right panel: relative difference between counts with and without extinction correction. Lower left panel: averaged extinction predicted by González et al. (2012) and Marshall et al. (2006) interstellar extinction model, for Galactic longitudes: $-10^{\circ}<\ell<0^{\circ}$ and $-20^{\circ}<$ $\ell<-10^{\circ}$, respectively; lower right panel: averaged extinction predicted by Nidever et al. (2012). In figures, we have considered a median within one degree in longitude.
González et al. (2012) and Marshall et al. (2006) for the same latitude ranges and adopting the median extinction for each pointing separated by one degree in longitude. As can be seen in Fig. 3 (lower left panel), averaged extinction in the Galactic plane reaches about $A_{V}=25$ mag with most values ranging from 15 to $20 \mathrm{mag}$. Generally, our averaged extinction variations in relative star counts are in agreement with those provided by González et al. (2012) and Marshall et al. (2006).

Since the star counts analysis is based on magnitude limited samples, an overestimated absorption will lead to overestimate star counts, because if the estimated extinction is too large, then stars that would fall beyond the magnitude cut-off would be overcorrected and thus be counted, biasing the corrected counts upward. Another effect is crowding. Since in-plane fields are more affected by crowding, here we will primarily lose the dimmer and redder stars than the ones that yield the high extinction values in off-plane fields.

This context provides an explanation for the awkwardly lower counts of the lowest latitude sample $\left(|b| \leq 0.375^{\circ}\right)$ in some ranges $\left(-5.0^{\circ}<\ell<-2.5^{\circ}\right.$ and $\left.\ell>-16.5^{\circ}\right)$, where the disk is expected to have higher counts, compared to the intermediate latitude sample $\left(0.375^{\circ}<|b| \leq 1.125^{\circ}\right)$. Comparison with the interstellar extinction distribution proposed by González et al. (2012) gives a hint that at $-5.0^{\circ}<\ell<-2.0^{\circ}$ the lower latitude extinction is underestimated, and the intermediate latitude range extinction is overestimated, giving rise to an extinction peak. Both effects naturally account for the apparently lower star counts of the lower latitude region compared to the mid-latitude region. The same reasoning applies to $\ell<-16.5^{\circ}$.

In any case, a significant increase in extinction for Galactic longitudes beyond (in the negative sense) $\ell \sim-14^{\circ}$ is appreciated for the three latitude ranges and are stronger for $|b| \leq$ $0.375^{\circ}$. The extinction grows until $\ell \sim-16^{\circ}$, decreasing towards $\ell \sim-20^{\circ}$ for the low latitude region, but shows a slight increase for the higher latitudes, see the discussion and references therein of Calbet et al. (1996) and González-Fernández et al. (2012).

The same feature was observed by LC01 in the regions towards long Galactic bar while comparing DENIS and CAIN data (their Fig. 8). The same authors also noticed the extra extinction from $\ell \sim-12^{\circ}$ to $\ell \sim-8^{\circ}$. The deeper VVV photometry in the present work allows a better definition of the feature, clearly displayed by very high extinction observed from $\ell \sim-11^{\circ}$ to $\ell \sim-6^{\circ}$.

In general the maps (Fig. 3, lower right panel) provided by Nidever et al. (2012) presents a good agreement with obtained ones by González et al. (2012), as well as with the one obtained by us. Some differences can be seen, such as the valley observed for Galactic latitudes $|b| \leq 1^{\circ}$, in our case for $\ell \sim-13.0^{\circ}$ and for $\ell \sim-12.0^{\circ}$ (Nidever et al. 2012) This can be attributed to the averaged extinction process used to elaborate the figure or even to the fact on using deep VVV data. To obtain the extinction from Nidever et al. (2012), we used the option all for stellar population and percentil for map type. The maps of Nidever et al. (2012) are available at their web page ${ }^{5}$.

Another point that should be considered in the comparison of our extinction method with the obtained ones by other authors is the point that we do not use local sources with $J-K_{\mathrm{s}}<0.5$, and the sources with $0.5<J-K_{\mathrm{s}}<1.0$ have no extinction correction applied to them. Despite all these points, we can see good agreement with our extinction estimate with other author. Detailed discussion about interstellar extinction distribution along the Galactic bar as well as analysis of 3D distribution is beyond the scope of the present paper.

It is important to mention that an aspect should be considered when comparing our data with the used ones by other author, for instance with Nidever et al. (2012, Fig. 1c), which is applicable to $(\ell, b)=\left(42^{\circ}, 0^{\circ}\right)$. The point is that for this direction, we are mainly observing disk stars, with a maximum density at distance of $6 \mathrm{kpc}$ from the Sun. This makes the population at low distances of Sun predominant at $K_{\mathrm{s}}=12$, with a higher ratio of dwarfs, which are bluer than $\left(J-K_{\mathrm{s}}\right)_{0}=1.0$. This is not our case (see color-magnitude diagrams in Paper I).

5 http://www.astro.virginia.edu/rjce/ 

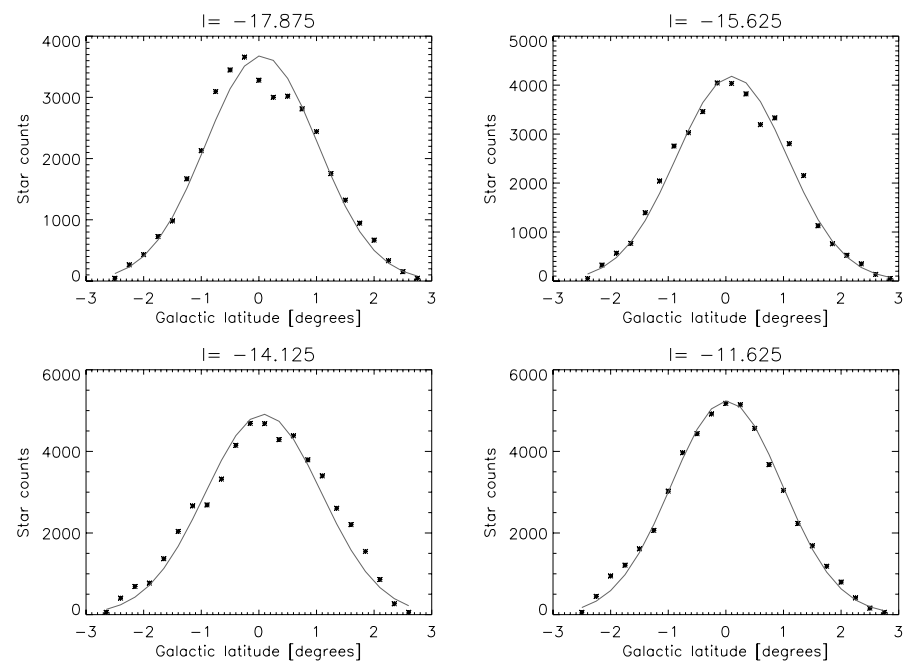

Fig. 4. Examples of Gaussian adjustments (lines) for the latitudinal profiles of the counts in $K_{\mathrm{s}}$ (asterisks) corrected by interstellar extinction effects. Galactic longitude is indicated at the top of the panels.

\subsection{Latitudinal counts}

Latitudinal star counts profiles provide valuable information on the structure of the Galaxy (Freudenreich et al. 1994). These profiles allow to infer the thickness of the dust-gas and stellar layers and their displacement with respect to the Galactic plane, among other applications. At the longitudes toward the Galactic anticenter, these profiles allow characterization of structures, such as the Galactic warp, among others. At the longitudes close to the Galactic center, they enable determining bar and bulge morphological parameters. Latitudinal profiles towards the long Galactic bar were built for the VVV+2MASS data, using extinction correction determined in this work (Sect. 3.1).

Gaussians were fitted (see some examples in Fig. 4) to the observed latitudinal profiles limited to $K_{\mathrm{s}} \leq 13.5 \mathrm{mag}$ and to colors $J-K_{\mathrm{s}} \geq 0.5 \mathrm{mag}$. The fits were done using an IDL version of a genetic algorithm called PIKAIA (Charbonneau 1995). Given that there are three parameters to be determined from a small volume of data, a small number of generations (100) with 60 populations each were adopted to insure a faster convergence. Twenty independent runs were performed, and the final solution is the median for the runs with $\chi^{2}$ within $1-\sigma$ of the distribution. The errors bars were determined from the standard deviation of the parameters. The merit function (a similar one was used by Larsen \& Humphreys 2003) used was

$\chi^{2}=\sum_{i=1}^{n}\left(N_{i}(\mathrm{obs})-N_{i}(\text { model })\right)^{2} / N_{i}(\mathrm{obs})$

where $N_{\text {model }}$ and $N_{\text {obs }}$ are the modeled and observed counts for each bin of Galactic latitude $(i)$.

It was obtained by adjusting the three parameters of the Gaussian function:

$f(z)=A \times \exp \left(-\left|z-z_{\mathrm{c}}\right|^{2} / K\right)$,

in which $K=2 \sigma^{2}$ is related to thickness, $A$ is the central surface density of the stars, and $z_{\mathrm{c}}$ the offset from the midplane. The range of parameters are presented in Table 2 .

As proposed by Cabrera-Lavers et al. (2007a), we also tried to adjust a $\operatorname{sech}^{2}$ function; however, Eq. (7) produced a better fit to our data. For the VVV+2MASS set, splitting it in $0.25^{\circ} \times 0.25^{\circ}$ regions gives 81 pointings with different longitudes, each one

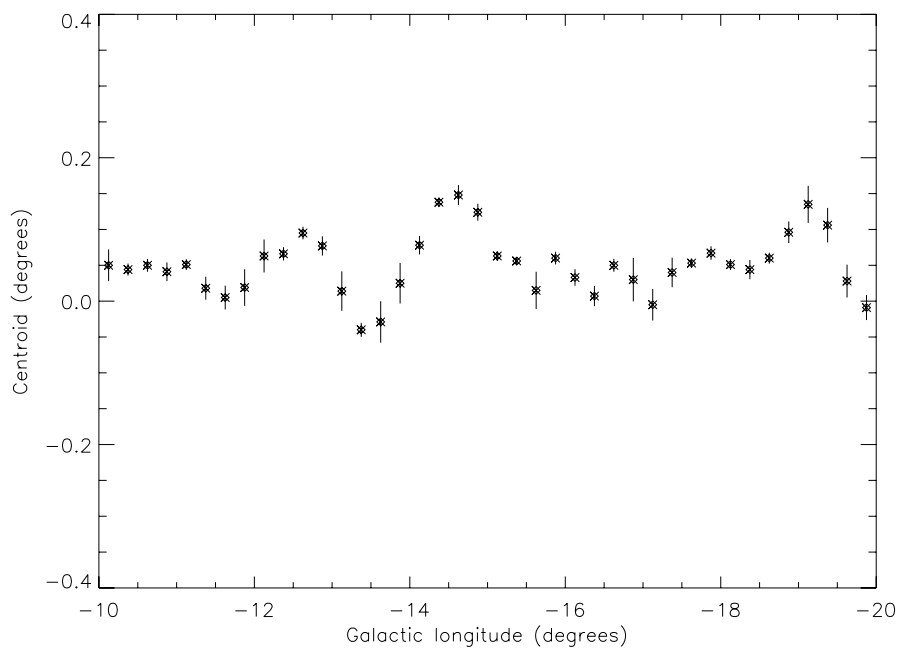

Fig. 5. Longitudinal profile for the stellar centroid variation obtained from adjustments of the latitudinal profiles of the counts $K_{\mathrm{s}}$.

Table 2. Range of parameters used to adjust the function represented by Eq. (7).

\begin{tabular}{lc}
\hline \hline Parameters & Range \\
\hline$z_{\mathrm{c}}$ & $-3.0+9.0 x_{1}$ \\
Width & $12.0 x_{2}$ \\
Heigth & maximum data height $\times 1.3 \times x_{3}$ \\
\hline
\end{tabular}

Notes. $x_{1}, x_{2}$, and $x_{3}$ are the normalization factor used by PIKAIA, ranging from 0.0 to 1.0 .

with 22 different latitude pointings. This sampling allows robust fits with good spatial resolution.

Figure 5 shows the variation in Gaussian centroids derived from the latitudinal profile fitting VVV+2MASS data. The variation is almost flat from $\ell \sim-11.5^{\circ}$ to $\ell \sim-10^{\circ}$, reaching a local minimum at $\ell \sim-11.5^{\circ}$ and increasing linearly to $\ell \sim-12.75^{\circ}$. After that, a decrease is seen until reaches a minimum at $\ell \sim-13.8^{\circ}\left(z_{\mathrm{c}}=-0.05^{\circ}\right)$. A significant increase is then seen reaching a maximum at $\ell \sim-14.5^{\circ}$, after that there is a small local decrease, and the centroid then remains roughly constant until $\ell \sim-18.5^{\circ}$.

We have restricted our analysis up to longitudes lower than $\ell \sim-10^{\circ}$ since many latitudinal profiles tend to have irregular shapes toward the bulge, which removes meaning from Gaussian fits. These irregularities could be due to inadequate interstellar extinction correction, or more likely, to VVV photometric incompleteness toward the more crowded Galactic center region. This is supported by the study of Amôres et al. (2012) aimed at detecting galaxies in the VVV database. The authors report that a closer examination reveals that many sources classified by VVV as extended objects are in fact composed of systems with two or three stars.

Freudenreich et al. (1994) analyzed latitudinal profiles for COBE/DIRBE emission, finding warp and flare structures toward the Galactic anti-center at $240 \mu \mathrm{m}$, which is dominated by dust emission. At NIR and MIR COBE/DIRBE wavelengths one can see the displacement in latitude of the brightness peak toward the Galactic center, but the resolution of their latitudinal profiles (each $10^{\circ}$ ) does not allow the bar region to be identified. On the other hand, the adjustment for the latitudinal profiles obtained by BL91 (their Fig. 12) shows clearly a tilt in the variation of the centroid of the surface brightness distribution, with a 
typical deviation of $\pm 0.4^{\circ}$ in latitude; unfortunately, their figure only shows the variation from Galactic longitudes $\left(|\ell|<10^{\circ}\right)$.

By adjusting data from the IRAS $100 \mu \mathrm{m}$ band that traces dust distribution (AL05) at one degree interval (Amôres 2005), found a centroid distribution compatible to obtaining one by BL91 with a significative decrease for longitudes lower than $\ell \sim-13^{\circ}$, as also found in the present work with VVV data. In fact, the asymmetric shape for the stellar distribution that can be seen in Fig. 5 suggests a similar feature for the dust distribution that dominates this region. Amôres (2005) found a variation of 0.3 degrees in $z_{\mathrm{c}}$ that is compatible with the expected scenario for regions of leading dust lanes. The author also analyzed latitudinal profiles for HI for specific ranges of velocities (belonging to peaks related to FIR emission) and a significant variation in the centroid is found toward $\ell \sim-15^{\circ}$. Making the assumption of non-circular movements at the Galactic center, a distance equal to $10.7 \mathrm{kpc}$ (considering $v \sim-110 \mathrm{~km} \mathrm{~s}^{-1}$ ) was found. Marshall et al. (2008) analyzed the latitudinal profiles for extinction obtained with the extinction model proposed by Marshall et al. (2006) and CO but for a longitude range of $10^{\circ}$ to $-4^{\circ}$.

From the latitudinal profile fits we obtained a parameter that we call to $K$ (see Eq. (7)), which is also useful in estimating the vertical thickness of the bar that can be estimated using the expression

$h_{z}=d \sin (\sigma)$,

where $d$ is the average distance for the points, e.g., $(\ell, b)$ pairs considered in this work to the far side of the Galactic bar, equal to approximately $10.0 \mathrm{kpc}$ (LC01). We get an average $\sigma=1.01 \pm 0.03$ degrees; hence we get a vertical thickness approximately equal to $176 \pm 6 \mathrm{pc}$. This value is higher than $100 \mathrm{pc}$ obtained by Cabrera-Lavers et al. (2007a) using red clump stars. First, we must take into account that we are using a wider range of stellar types than red clumps, and we are also observing the bar with a different angle (at positive longitudes, we observe the bar almost perpendicularly, whereas at negative longitudes our line of sight is more tangential to the bar). More importantly, in our star counts variations, we also include variations from the disk + bulge + bar instead of the isolated bar, therefore we get a higher thickness which is the average of the three embedded structures with their corresponding weights. A rough estimation of the bar thickness might be given considering that $\sigma=1.01 \pm 0.03^{\circ}$ is the $\sigma$ of the sum of two Gaussians ${ }^{6}$. We know that the contribution of the disk + bulge gives a $\sigma_{b+d} \approx 2.0^{\circ}$ at $\ell \sim-12^{\circ}$ (López-Corredoira et al. 2004, 2005), and roughly bulge + disk gives the same counts as the bar (see Fig. 3, and compare the inplane counts with counts at $b=2^{\circ}$ ); hence, $\sigma_{\text {bar }}=0.7^{\circ}$, so the vertical thickness of the bar alone should be around 120 pc, closer to Cabrera-Lavers et al. (2007a) results. We state that bulge + disk have roughly the same counts at $\ell=12^{\circ}, b=0^{\circ}$ as the bar, because at $b=1.75^{\circ}$ (where the bar contribution is negligible), they are 2.5 times lower (instead of 1.46 times lower expected from the Gaussian of bulge+disk) attributing the difference to the bar.

\section{Discussion}

Figure 6 shows the counts up to $K_{\mathrm{s}}=13.5 \mathrm{mag}$ for the long bar region with and without extinction correction, as well as for the

\footnotetext{
6 If we have a Gaussian for the bar with $\sigma_{\text {bar }}=0.7$ and a second Gaussian for the disk + bulge with $\sigma_{b+d}=2.0$ with the same amplitude, the sum of both functions approximately gives a Gaussian with $\sigma_{\text {total }}=1.01$.
}

residual differences. In Fig. 6 (upper panel), a structure ending toward $\ell \sim-8^{\circ}$ is seen that can be attributed to the Galactic bulge. A long bar structure can be seen as the green region extending up to $\ell \sim-14^{\circ}$. For longitudes after $\ell \sim-15^{\circ}$, the stellar density decreases as illustrated by the lighter blue area in Fig. 6 (upper panel). In this figure, part of stars are red giants. The far side of the Galactic bar as presented in this paper has an end at $\ell \approx-14^{\circ}$, and this is not only due to the high extinction observed after bar ends but also the density of stars that falls considerably in this longitude as seen from several surveys in the NIR and MIR.

Moreover, works on Galactic resonances (Amaral \& Lépine 1997; Lépine et al. 2001; Mishurov et al. 2009, and references therein) explain how regions toward them, such as the end of the Galactic bar, suffer the effects of corotation. In these regions, there is also smaller quantity of gas and stars. Briefly, the effects of these resonances can be understood as follows. The gas dynamics in the perturbed potential of the spiral arms is such that inside the corotation radius, a net flow is produced toward the center. Beyond corotation, a net flow is produced toward the external parts of the disk. This dynamics results in a pumping gas out from the corotation region (Amôres et al. 2009).

Figure 6 (middle panel) clearly illustrates the importance of correcting for extinction. Without it, the structure identified in Fig. 6 (upper panel) is not observed. The star counts decrease too quickly at $\ell \sim-8^{\circ}$, and the structure is too thick for $\ell>$ $-8^{\circ}$ and too thin for $\ell<-8^{\circ}$. Furthermore, the counts without extinction correction produce a shape without the symmetry seen in Fig. 6 (upper panel). In Fig. 3, we pointed that there is a clear decrease in the counts until $\ell \sim-14^{\circ}$. Certainly, the smoothing in longitude and latitude decreases the contrast in Fig. 3, and this is better observed in Fig. 6 (upper panel).

Figure 6 (lower panel) shows a map for the relative difference (see Sect. 4.1) in counts between the upper panels, which roughly corresponds to an extinction map. In this figure, the high extinction structures toward the end of the long Galactic bar are highlighted. LC01 argue that longitudes corresponding to the negative limit of the bar are at almost twice the distance relative to the center as those on the nearest side, which are also affected by higher extinction. This feature can also be seen in the comparison with GLIMPSE data shown in Fig. 7.

The difference between $m_{K_{\mathrm{s}}}$ and $m_{K_{\mathrm{s}} \text { correc }}$ is proportional to the average extinction (see Eq. (1)); there is only dependence on the structure distance through the distribution of dust and stars along the line of sight. Therefore, Fig. 6 (lower) is a map of average extinction until the distance of the average position of the stars along the line of sight. It is also possible to identify an asymmetry in the distribution of interstellar extinction with respect to the formal Galactic plane from $\ell \sim-16^{\circ}$ to $\ell \sim-14^{\circ}$. This feature was also identified in IRAS $100 \mu \mathrm{m}$ (Amôres 2005).

As found by LC01 (and discussed in Sect. 4.1), we also identify high extinction toward $-10^{\circ}<\ell<-8^{\circ}$ in our VVV+2MASS data. The extinction decreases until the bar ends at $\ell \sim-14^{\circ}$, which can be interpreted as the dust lanes mentioned by Calbet et al. (1996), Rodriguez-Fernandez et al. (2006), Nagai et al. (2007), Marshall et al. (2008), and Paper I. Then, a high extinction region extends up to $\ell<-19^{\circ}$ in the proximity of the $3 \mathrm{kpc}$-arm. This latter high extinction feature contrasts with the star counts density in Fig. 3 (upper right panel).

Concerning the near side of the Galactic bar, several works have placed it at $\ell \sim+27^{\circ}$, approximately $5.7 \mathrm{kpc}$ from the Sun, as pointed out by Hammerseley et al. (1994, 2000, e.g. H94, 


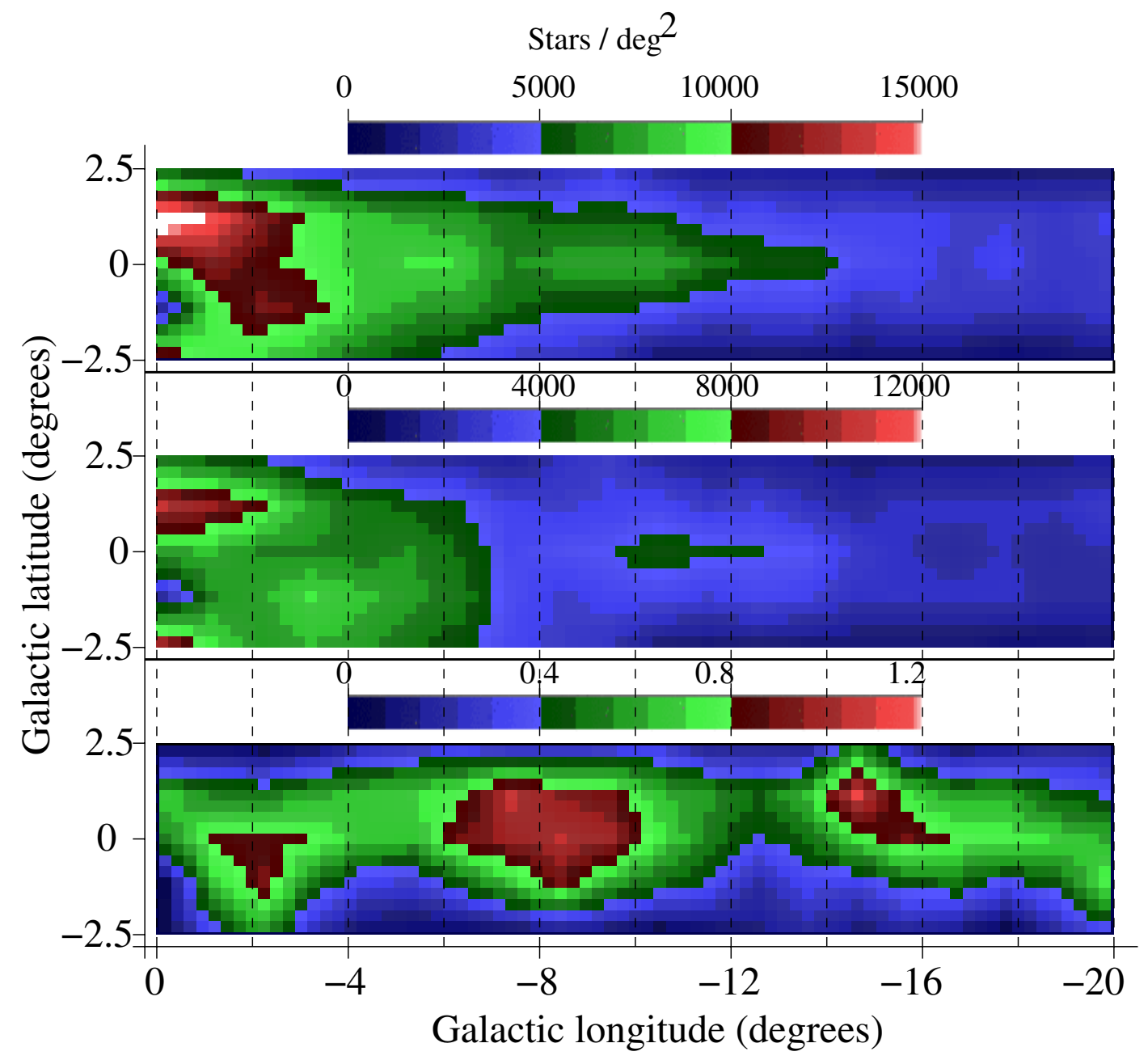

Fig. 6. VVV counts (up to $K_{\mathrm{s}}=13.5 \mathrm{mag}$ ) towards the long Galactic bar: corrected for interstellar effects (upper panel); without correction (middle panel); relative difference (lower panel), star counts binned $\left(\Delta l=\Delta b=1^{\circ}\right)$ and interpolated.

H00), Garzón (1993), LC01, Cabrera-Lavers et al. (2007a), and Picaud et al. (2003), among others.

We have adopted distance to the far side of Galactic bar of $11.1 \mathrm{kpc}$ (as also used by LC01). We also argue that the long bar tip on the far side is at longitude limit of $\ell \approx-14^{\circ}$. If we consider a semi-major axis length $L_{0}$ of $4.0 \pm 0.5 \mathrm{kpc}$, values that are compatible with the ones obtained by LC01, H00, H94, among others, we can estimate the inclination angle $(\alpha)$ using

$\alpha=\ell+a \sin \left[\left(R_{0} / L_{0}\right) \sin (\ell)\right]$,

where $R_{0}=8.0 \mathrm{kpc}$ is the distance from the Sun to the Galactic center, $L_{0}$ is the semi-major axis length and $\ell$ is the longitude that corresponds to the end of bar.

We can obtain $\alpha$ by considering the error for both $\ell$ and $L_{0}$. We assume that $\ell \approx-14^{\circ}$ and $L_{0}=4.0 \pm 0.5 \mathrm{kpc}$. Using Eq. (9) we obtained $\alpha=43^{\circ} \pm 5^{\circ}$. This angle is the same one as the measured values in the positive longitudes (e.g. H00), so we conclude the bar must be straight.

This value agrees with other authors such as Peters (1976), Nakai (1992), Hammersely et al. (2000), and Sevenster et al. (1999), who report $45^{\circ}, 43^{\circ}$, and $45^{\circ}$, respectively. This value also agrees with findings pointed out by LC01 $\left(43.0^{\circ}\right)$ and later by several authors, e.g., LC07 and references therein; Cabrera-Lavers et al. (2007a,b), Benjamin et al. (2005), Vallenari et al. (2008) report an angle of $45^{\circ}$. A schematic view of bar representation can be seen in Fig. 8 of Paper I. Figure 7 (middle panel) shows the counts at [8.0] $\mu \mathrm{m}$ with a cut-off at $11.0 \mathrm{mag}$ and corrected for extinction using the relations provided by M11. Determination of the extinction correction made use of the deep combined VVV+2MASS+GLIMPSE data set, as described in Sect. 2.2.

A structure, coded in darker green and seen reaching $\ell \sim$ $-13^{\circ}$, corresponds to the long Galactic bar and is then followed by a region having smaller and almost constant star counts. As in Fig. 6, the bulge is clearly seen, ending toward $\ell \sim-8^{\circ}$. Figure 7 (upper panel) shows the map using the extinction correction provided by M11 using only the less deep 2MASS+GLIMPSE combined data (no VVV).

Benjamin et al. (2005) used GLIMPSE data at [4.5] $\mu \mathrm{m}$, to derive an inclination angle of $44 \pm 10^{\circ}$ for the bar, with $R_{\text {bar }}=4.5 \pm 0.5 \mathrm{kpc}$. As pointed by LC07 their general results agrees with those obtained by LC01, also claiming for a long bar. Their longitudinal source-density profiles at [4.5] $\mu \mathrm{m}$ show a decrease in the counts at $\ell \sim-13^{\circ}$. They also produced a star counts map in which one can identify some structures similar to those reported in this work. However, their picture is not as sharp as our Fig. 7 (middle panel), which we think is due to our improved extinction correction using deep $J$ and $K_{\text {s }}$ photometry and to extinction being lower at [8.0] $\mu \mathrm{m}$. A marked difference between both maps is that Benjamin et al. (2005) found significantly fewer stars at Galactic longitudes from $\ell \sim-17^{\circ}$ to $-15^{\circ}$, a region that we point out as a high extinction zone. 

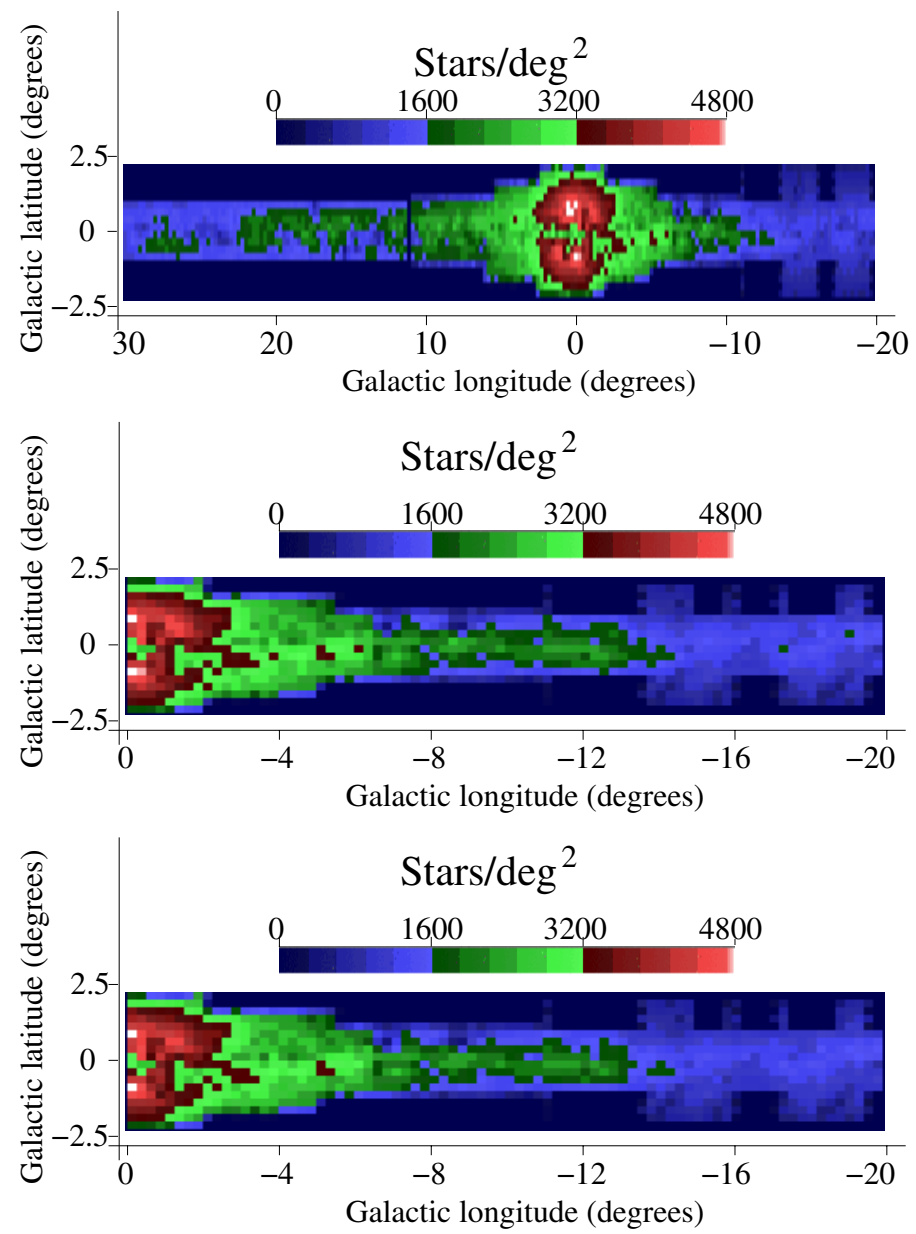

Fig. 7. GLIMPSE counts (up to [8.0] $\mu \mathrm{m}=11.0 \mathrm{mag}$ ) using extinction method presented in Eq. (4) that uses the combined data (upper and middle panel). Upper panel: GLIMPSE+2MASS. Middle panel: GLIMPSE+VVV+2MASS with interstellar extinction correction going deeper in the comparisons as presented in Table 1. Lower panel: GLIMPSE+VVV+2MASS without interstellar extinction correction.

In Fig. 2 of Nidever et al. (2012), there is some gap after $\ell \sim-15^{\circ}$, although it is not very clear, probably because 2MASS data is not deep enough on the range of magnitudes used. However, the features of the Galactic bar can be seen in GLIMPSE data, when observed considering appropriate range of magnitudes from $\sim 14$ to $\sim 6.5$, see for instance maps in Churchwell et al. (2009) and Benjamin et al. (2005) as mentioned in the last paragraph.

Figure 7 (upper panel) shows the counts at [8.0] $\mu \mathrm{m}$ but only based on 2MASS photometry instead of combined VVV+2MASS data. It can be seen that the structure of the long bar ends towards $\ell \sim-11^{\circ}$, unlike the limit obtained when based on the combined data, thus allowing for a deeper analysis of the Galactic bar. Figure 7 (lower panel) shows the counts [8.0] $\mu \mathrm{m}$ that are almost similar to the one presented in Fig. 7 (middle). The similarity between the counts highlight that extinction is much lower at [8.0] $\mu \mathrm{m}$, as discussed in Sect. 3.2 (see Eq. (4)).

This can be visualized better in Fig. 8, which shows longitudinal profiles for counts at [8.0] $\mu \mathrm{m}$ using only GLIMPSE+ 2MASS and combined GLIMPSE+2MASS+VVV data. There are two main aspects to be considered by this figure: i) the number of sources clearly increases with the addition of VVV data since there are more matches to GLIMPSE sources that were not detected by 2MASS (see Table 1); ii) some differences in the

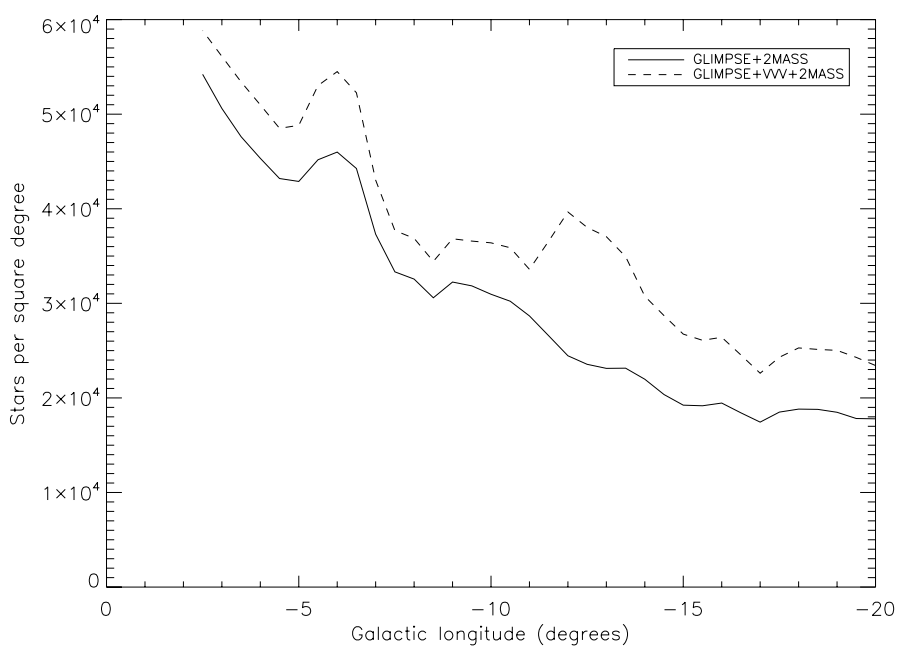

Fig. 8. Longitudinal profile for GLIMPSE counts in [8.0] $\mu \mathrm{m}$ using the extinction method presented in Eq. (4) that uses the combined data.

shape of profile (most for $\ell<-10^{\circ}$ ) can also be noted and attributed to the use of extinction correction with more stars. The relative count differences attributed by combining the VVV data are approximately $20 \%$ and $27 \%$ for $\ell \geq-10^{\circ}$ and $\ell<-10^{\circ}$, respectively. A structure with a double peak can be seen within the longitude range: $-14^{\circ}<\ell<-12^{\circ}$. For $\ell<-14^{\circ}$ a significant decrease in the counts was independently found by LC07 using MSX data at [8.7] $\mu \mathrm{m}$.

Concerning another study of star counts toward the far side of long bar; Benjamin et al. (2005) found using GLIMPSE at [4.5] $\mu \mathrm{m}$, approximately $2.0 \times 10^{4}$ sources per square degree. LC01 find counts for $m_{K} \leq 9.0$ of approximately 2000 using DENIS data averaged over $\Delta \ell=5^{\circ}$. We can estimate the counts expected by a model, in this case using a similar model as proposed by LC01 with disk density given by López-Corredoira et al. (2002, 2004), with the luminosity function proposed by Eaton et al. (1984) and the counts in the Galactic bar based on the density distribution proposed by López-Corredoira et al. (2007) with the luminosity function considered as an average of the disk and bulge luminosity function. We have obtained counts in good agreement with ones observed by VVV. For instance, for $\ell \sim-15.0^{\circ}$ for two ranges of Galactic latitudes, $0.375^{\circ}<|b| \leq 1.125^{\circ}$ and $1.375^{\circ} \leq|b| \leq 2.125^{\circ}$, we estimate $10^{4}-10^{5}$ sources per square degree (considering as magnitude limit $K_{\mathrm{s}}=13.5 \mathrm{mag}$ ), respectively. The values of parameters used in the model are in good agreement with those ones obtained in this work.

\section{Conclusions}

In this paper we have used deep VVV photometric data to perform a detailed analysis of the far side of long Galactic bar, which is the less studied side, by far, in the literature. This analysis was based on star counts in the $K_{\mathrm{s}}$ band for the region extending from $\ell \sim-20.0^{\circ}$ to $0.0^{\circ}$ and for Galactic latitudes $|b| \leq 2^{\circ}$.

We used the extinction correction recipes provided by López-Corredoira et al. (2001) and Majewski et al. (2011) on combined VVV+2MASS and GLIMPSE data, respectively. Since there is more extinction toward negative longitudes than toward positive longitudes (LC01), the average extinction correction methods employed in this work has the advantage of using many distant stars with high $J-K_{\mathrm{s}}$ and $H$ values, as provided by VVV survey. 
Even though the method used in this work had been presented and used in other works, we expanded it by comparing it with other more complexes extinction models, as well as with star counts simulations as presented in Appendix A.

The use and combination of extinction methods with VVV data allow us to better account for the effects of interstellar extinction on star counts for both the $K_{\mathrm{s}}$ and [8.0] $\mu \mathrm{m}$ bands. An extinction map was produced (Fig. 6, lower panel), revealing high extinction regions along the bar, more notably slightly farther than the bar end $\left(\ell \sim-14^{\circ}\right)$. A tilt in the dust distribution (Fig. 6, lower panel) is noted, observed towards $\ell \sim-15^{\circ}$ $\left(b \sim 0.5^{\circ}\right)$ with similar characteristics to those observed at IRAS $100 \mu \mathrm{m}$ (Amôres 2005), which is a feature not observed, for instance, in the Marshall et al. (2006) extinction maps.

Even though this work was not devoted to the study of interstellar extinction, our results highlight the potential use of the VVV survey for modeling the dust distribution for both 2D and 3D maps/models as presented by González et al. (2012) and Chen et al. (2013). This type of analysis also has the potential for studying interstellar extinction in the Galactic disk with the use of NIR and MIR colors using combined VVV and GLIMPSE data.

We modeled the extinction in the Galactic bar region more accurately and with higher resolution through using VVV data and more importantly, combined this with 2MASS and GLIMPSE data. In the first case, we complemented VVV observations for brighter sources and, in the second used MIR colors that allow a more precise determination of extinction. The extinction model used should not significantly change our bar representation (Figs. 6 and 7). The impact of any other extinction model should only appear on a small scale as an effect of the resolution not modifying the large structure of long bar as presented in this work. Even if other extinction laws were considered the normalization of the counts would be affected, but the shape would be similar.

In terms of the parameters of the Galactic bar, our results support those of previous studies (H00, LC01, LC07 and references therein). But the spatial description of the long Galactic bar as presented in this work (Figs. 6 and 7 lower panels) surpasses what has been known previously, and allows us to better identify its structure, not only in resolution but also in completeness. Nevertheless, previous works by other authors have allowed us to clearly identify structures along the bar, and this has allowed us to identify and compare structure and regions. For instance, in the region from $\ell \sim-14.0^{\circ}$ to $-12.0^{\circ}$ that bar was possibly first detected reported by LC01. However, the limits shown in this work are now much more clearly defined than in this or any other previous study, using data from the DENIS, CAIN and 2MASS surveys. The same goes for all negative bar regions.

In the same way, with resulting longitudinal profiles made with resolution (keeping robustness) at 1 degree interval, instead of 5 degrees as in previous works in the NIR, we more clearly reveal a structure extending to $\ell \sim-14^{\circ}$. Our work can also be used to constrain parameters by using models that separate bulge and bar counts. The latitudinal profiles allow us to obtain the centroid (as well as the bar's vertical thickness) variation of the stellar distribution with longitude in the $K_{\mathrm{s}}$ band that shows a clear feature with a minimum at $\ell \approx-13.8^{\circ}$.

Acknowledgements. We thank both the referee Dr Chris Flynn and the anonymous referee for useful, valuable, and detailed comments on the manuscript that also improved the paper's clarity. We gratefully acknowledge use of data from the ESO Public Survey program ID 179.B-2002 taken with the VISTA telescope, data products from the Cambridge Astronomical Survey Unit, and funding from the FONDAP Center for Astrophysics 15010003 , the BASAL CATA Center for Astrophysics and Associated Technologies PFB-06, the MILENIO Milky Way Millennium Nucleus from the Ministry of Economy's ICM grant P07-021$\mathrm{F}$, and the FONDECYT from CONICYT. Eduardo Amôres obtained financial support for this work from the Fundação para a Ciência e Tecnologia (FCT) under the grants SFRH/BPD/42239/2007 and PTDC/CTE-SPA/118692/2010 and also CNPq (311838/2011-1). He also acknowledges the hospitality and courtesy during his visit to the IAC, as well as for the partial support for that. EBA thanks the SIM-GRIDPT computing center funded under project REDE/1522/RNG/2007. This work also made use of the computing facilities of the Laboratory of Astroinformatics (IAG/USP, NAT/Unicsul), where the cost was covered by the Brazilian agency FAPESP (grant 2009/54006-4) and the INCT-A. M.L.C. was supported by the grant AYA2007-67625-CO2-01 of the Spanish Science Ministry. CGF gratefully acknowledges the funding from the Spanish Ministerio de Ciencia e Innovación (MCINN) under AYA2010-21697C05-5 and the Consolider-Ingenio 2010 Program grant CSD2006-00070: First Science with the GTC (http://www.iac.es/consolider-ingenio-gtc). This publication made use of data products from the Two Micron All Sky Survey, which is a joint project of the University of Massachusetts and the Infrared Processing and Analysis Center/California Institute of Technology, funded by the National Aeronautics and Space Administration and the National Science Foundation. This work is based in part on observations made with the Spitzer Space Telescope, which is operated by the Jet Propulsion Laboratory, California Institute of Technology under a contract with NASA.

\section{References}

Alard C. 2001, A\&A 379, L44

Amaral, L. H., \& Lépine, J. R. D. 1997, MNRAS 286, 885

Amôres, E. B. 2005, Ph.D. Thesis, Universidade de São Paulo

Amôres, E. B., \& Lépine, J. R. D. 2005, AJ, 130, 659 (AL05)

Amôres, E. B., Lépine, J. R. D., \& Mishurov, Yu. N. 2009, MNRAS, 400, 1768

Amôres, E. B., Sodré, L., Minniti, D., et al. 2012, AJ, 144, 127

Babusiaux, C., Gómez, A., Hill, V., et al. 2010, A\&A, 519, A77

Benjamin, R. A., Churchwell, E., Babler, B. L., et al. 2003, PASP, 115, 953

Benjamin, R. A., Churchwell, E., Babler, B. L., et al. 2005, ApJ, 630, L149

Blitz, L., \& Spergel, D. N. 1991, ApJ, 379, 631 (BL91)

Burton, W. B., \& Liszt, H. S. 1983, A\&AS, 52, 63

Calbet, X., Mahoney, T., Hammersley, P. L., Garzon, F., \& López-Corredoira, M. 1996, ApJ, 457, L27

Cabrera-Lavers, A., Hammersley, P. L., González-Fernández, C., et al. 2007a, A\&A, 465, 825

Cabrera-Lavers, A., Bilir, S., Ak, S., Yaz, E., \& López-Corredoira, M. 2007b, A\&A, 464, 565

Churchwell, E., Babler, B. L., Meade, M. R., et al. 2009, PASP, 121, 213

Charbonneau, P. 1995, ApJS, 101, 309

Chen, B. Q., Schultheis, M., Jiang, B. W., et al. 2013, A\&A, 550, A42

Dame, T. M., Elmegreen, B. G., Cohen, R. S., \& Thaddeus, P. 1986, ApJ, 305, 892

Eaton, N., Adams, D. J, \& Giles, A. B. 1984, MNRAS, 208, 241

Freudenreich, H. T., Berriman, G. B., Dwek, E., et al. 1994, ApJ, 429, L29

Gardner, E., \& Flynn, C. 2010, MNRAS, 405, 545

Garzón, F., Hammersley, P. L., Mahoney, T., et al. 1993, MNRAS, 264, 773

Glass, I. S. 1999, Handbook of Infrared Astronomy (Cambridge: Cambridge Univ. Press)

González, O. A., Rejkuba, M., Minniti, D., et al. 2011, A\&A, 534, L14

González, O. A., Rejkuba, M., Zoccali, M., et al. 2012, A\&A, 543, A13

González-Fernández, C., López-Corredoira, M., Amôres, E. B., et al. 2012, A\&A, 546, A107 (Paper I)

Habing, H. J., Sevenster, M. N., Messineo, M., van de Ven, G., \& Kuijken, K. 2006, A\&A, 458, 151

Hammersley, P. L., Garzón, F., Mahoney, T., \& Calbet, X. 1994, MNRAS, 269, 753 (H94)

Hammersley, P. L., Garzón, F., Mahoney, T. J., López-Corredoira, M., \& Torres, M. A. P. 2000, MNRAS, 317, L45 (H00)

Hayakawa, S., Matsumoto, T., Murakami, H., et al. 1981, A\&A, 100, 116

Larsen, J. A., \& Humphreys, R. M. 2003, AJ, 125, 1958

Lépine, J. R. D., Mishurov, Yu., \& Dedikov, Y. 2001, ApJ, 546, 234

Lizst, H. S., \& Burton, W. B. 1980, ApJ, 236, 779

López-Corredoira, M., Hammersley, P. L., Garzón, F., et al. 2001a, A\&A, 373, 139 (LC01)

López-Corredoira, M., Garzón, F., \& Hammersley, P. L. 2001b, MNRAS, 320, 31

López-Corredoira, M., Cabrera-Lavers, A., Garzón, F., \& Hammersley, P. L. 2002, A\&A, 394, 883 
López-Corredoira, M., Cabrera-Lavers, A., Gerhard, O., \& Garzón, F. 2004, A\&A, 421, 953

López-Corredoira, M., Cabrera-Lavers, A., \& Gerhard, O. E. 2005, A\&A, 439, 107

López-Corredoira, M., Cabrera-Lavers, A., Mahoney, T. J., et al. 2007, AJ, 133, 154 (LC07)

López-Corredoira, M., Cabrera-Lavers, A., González-Fernández, C., et al. 2011 [arXiv: 1106.0260]

Marshall, D. J., Robin, A. C., Reylé, C., Schultheis, M., \& Picaud, S. 2006, A\&A, 435, 635

Marshall, D. J., Fux, R., Robin, A. C., \& Reylé, C. 2008, A\&A, 477, L21

Majewski, S., Zasowski, G., \& Nidever, D. 2011, ApJ, 739, 25 (M11)

Matsumoto, T., Hayakawa, S., Koizumi, H., et al. 1982, in The galactic center; Proc. Workshop, Pasadena, CA, January 7, 8, 1982 (A83-40676 19-90) (New York: American Institute of Physics), 48

Martínez-Valpuesta, I., \& Gerhard, O. 2011, ApJ, 734, L20

Minchev, I., Nordhaus, J., \& Quillen, A. C. 2007, ApJ, 664, L31

Minniti, D. 1996, ApJ, 459, 175

Minniti, D., Lucas, P. W., Emerson, J. P., et al. 2010, New Astron., 15, 433

Mishurov, Yu. N., Amôres, E. B., \& Lépine, J. R. D. 2009, Physics-Doklady, 54, 466

Nagai, M., Tanaka, K., Kamegai, K., \& Oka, T. 2007, PASJ, 59, 25

Nakai, N. 1992, Astron. Soc. Japan, 44, L27

Nidever, D., Zasowski, G., \& Majewski, S. R. 2012, ApJS, 201, 35

Nishiyama, S., Nagata, T., Baba, D., Haba, Y., et al. 2005, ApJ, 621, L105

Paczynski, B., Stanek, K. Z., Udalski, A., et al. 1994, ApJ, 435, L113

Peters, W. L. 1975, ApJ, 195, 617
Picaud, S., Cabrera-Lavers, A., \& Garzón, F. 2003, A\&A, 408, 141

Popowski, P., Griest, K., Thomas, C. L., et al. 2005, ApJ, 631, 879

Rattenbury, N. J., Mao, S., Sumi, T., \& Smith, M. C. 2007, MNRAS, 378, 1064

Rieke, G. H., \& Lebovsky, M. J. 1985, ApJ, 288, 618

Robin, A. 2009, A\&A, 500, 165

Robin, A. C., Marshall, D. J., Schultheis, M., \& Reylé, C. 2012, A\&A, 538, A106

Rodriguez-Fernandez, N. J., Combes, F., Martin-Pintado, J., Wilson, T. L., \& Apponi, A. 2006, A\&A, 455, 963

Romero-Gómez, M., Athanassoula, E., Antoja, T., \& Figueras, F. 2011, MNRAS, 418, 1176

Saito, R. K., Zoccali, M., McWilliam, A., et al. 2011, AJ, 142, 76

Saito, R. K., Hempel, M., Minniti, D., et al. 2012, A\&A, 537

Sarajedini, A., Lee, Y.-W., \& Lee, D.-H., et al. 1995, ApJ, 450, 712

Sevenster, M., Prasenjit, S., Valls-Gabaud, D., \& Fux, R. 1999, MNRAS, 307, 584

Sellwood, J. 1981, A\&A 99, 362

Skrutskie, M. F., Cutri, R. M., Stiening, R., et al. 2006, AJ, 131, 1163

Stanek, K. Z., Mateo, M., Udalski, A., et al. 1994, ApJ, 429, L73

Vallenari, A., Ragaini, S., \& Bertelli, G. 2008, in: Formation and Evolution of Galaxy Bulges, eds. M. Bureau, E. Athanassoula, \& B. Barbuy (Cambridge: Cambridge Univ. Press), IAU Symp., 245, 371

Vanhollebeke, E., Groenewegen, M. A. T., \& Girardi, L. 2009, A\&A, 498, 95

Wainscoat, R. J., Cohen, M., Volk, K., Walzer, H. J., \& Schwartz, D. E. 1992, ApJS, 83, 111

Weiland, J. L., Arendt, R. G., Berriman, G. B., et al. 1994, ApJ, 425, L81

Pages 13 to 14 are available in the electronic edition of the journal at http://www . aanda. org 


\section{Appendix A: Testing the method of extinction correction for the star counts}

In Sect. 3.1, we have proposed a simple algorithm to correct the $K_{\mathrm{s}}$-band star counts from extinction, in which we only need the observed magnitudes in $J$ and $K_{\mathrm{s}}$. There is some discussion of the method in the literature (e.g., Alard 2001; López-Corredoira et al. 2001) or a similar method for redder wavelengths (e.g., M11), and the logic is clear: the average reddening is proportional to the extinction, thus the average $\left(J-K_{\mathrm{s}}\right)$ gives us information about the average extinction along the line of sight. In our case, looking at the central regions $\left(|\ell| \leq 20^{\circ},|b|<2.5^{\circ}\right)$ and excluding the sources with $\left(J-K_{\mathrm{s}}\right)<0.5$, the contribution of disk sources is low. So, star counts are dominated by sources from the long bar or bulge+long bar. Since these structures have low dispersion of distances, the dispersion of extinction of the sources will not be high; as a result the application of the method of correction will recover more or less the average counts up to a limiting magnitude. In this section, we carry out some simulations to show that the method of correction approximately recovers the equivalent counts if we have no extinction.

For our simulation, we take a model of the Galaxy with a stellar density of a disk from López-Corredoira et al. (2004), a bulge from López-Corredoira et al. (2005, model 2), and a long bar from LC07. Spiral arms and halo contributions are negligible so we do not include them here. In Fig. A.1, we plot the face-on image of the Galaxy according to this model. It is not important here whether this is a true representation of the Galaxy or not. At present, it is just a density distribution to test our method of extinction correction.

We use a model of populations from Wainscoat et al. (1992) to characterize the distribution of magnitudes and colors in each point of the space. We take the disk populations, since the differences with the older population of the bulge only affects very bright giants or supergiants (López-Corredoira et al. 2005), which are brighter than our range of magnitudes. The long bar is supposed to be an intermediate population, so again a distribution of populations like the disk is appropriate. Nonetheless, we insist that this is just a toy model for evaluating the method of extinction correction, so variations in this assumption are not important here. With the mentioned density distribution and the population distribution, we integrate along the line of sight (there is a factor $r^{2}$ in the integration), and we get the synthetic colormagnitude diagram of Fig. A.2 (left) at $\ell=-10^{\circ}, b=1^{\circ}$. We introduce a toy model of extinction. We assume a distribution of dust as

$\rho_{\text {dust }}=A_{\mathrm{d}} \exp \left(-\frac{R}{h_{R}}-\frac{|z|}{h_{z}}\right)$,

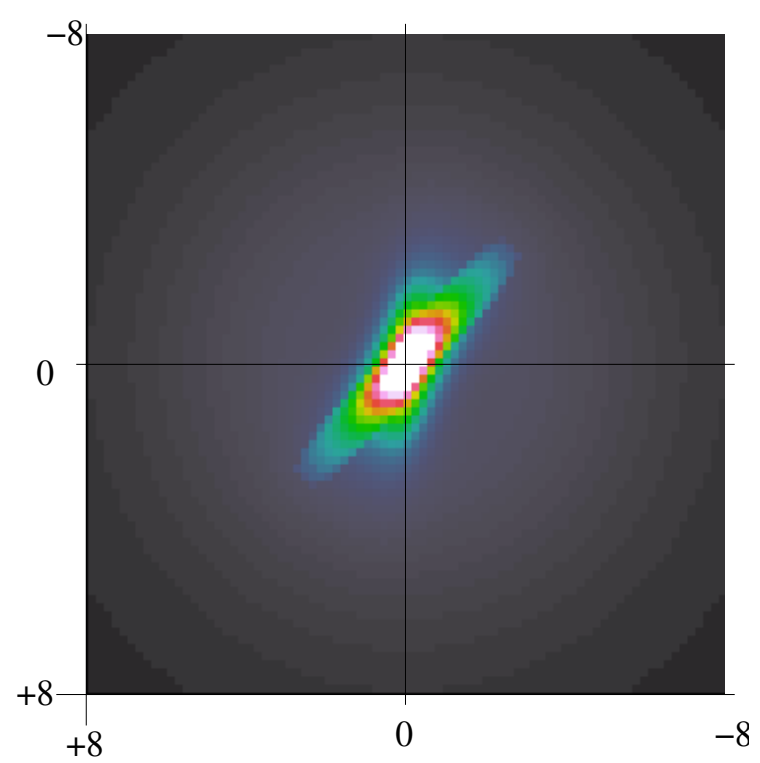

Fig. A.1. Face-on view (in units of kpc) of the Model of the Galaxy (see text in Appendix A) used to test the extinction correction method.

where $h_{R}=3 \mathrm{kpc}, h_{z}=0.1 \mathrm{kpc}$, and extinction in $K_{\mathrm{s}}$ in the solar neighborhood of $0.07 \mathrm{mag} / \mathrm{kpc}$, which gives $2.8 \mathrm{mag}$ of extinction up to the center of the Galaxy at distance $8.0 \mathrm{kpc}$. Again, whether this distribution of dust is exact or not is not important for our exercise. In Fig. A.2 (right) we plot the same color-magnitude diagram as before but introducing this extinction, assuming $\frac{A_{K_{\mathrm{s}}}}{A_{J}-A_{K_{\mathrm{s}}}}=\frac{3}{5}$ (see 5th paragraph of Sect. 3.1).

Now, we calculate the star counts with $\left(J-K_{\mathrm{s}}\right) \geq 0.5$ without extinction in Fig. A.2 (left) and with extinction (Fig. A.2. right) applying the correction of Eq. (A.1) with $\left(J-K_{\mathrm{s}}\right)_{0}=1.0$. The results are plotted in Fig. A.3, for three different lines of sight with three different total extinctions up to the semiaxis of the long bar: respectively $2.78 \mathrm{mag}$ up to a distance of $8 \mathrm{kpc}$ for $\ell=0, b=0 ; 1.24 \mathrm{mag}$ up to at distance $9.7 \mathrm{kpc}$ for $\ell=-10^{\circ}$, $b=1^{\circ} ; 0.47 \mathrm{mag}$ up to a distance $12.9 \mathrm{kpc}$ for $\ell=-20^{\circ}, b=2^{\circ}$. We therefore test different longitudes, latitudes, and extinctions.

As we can see in Fig. A.3, the method approximately recovers the counts that would be produced without extinction, within small errors typically of 5-10\%, which are not important for our purposes. The variation of $\frac{A_{K_{\mathrm{S}}}}{A_{J}-A_{K_{\mathrm{S}}}}=\frac{3}{5}$ will also introduce some extra error. 
A\&A 559, A11 (2013)
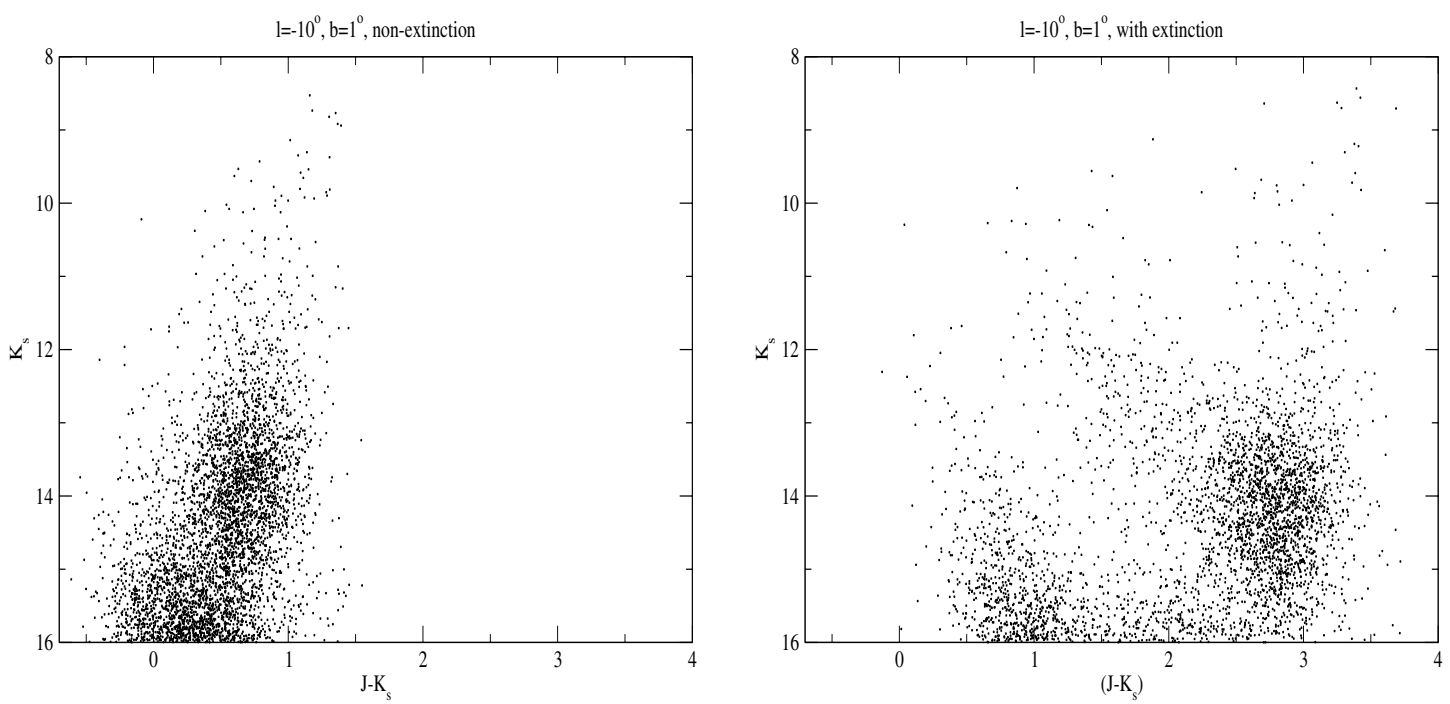

Fig. A.2. Synthetic color-magnitude diagram in the direction $\ell=-10^{\circ}, b=1^{\circ}($ left $)$ without extinction; (right) with extinction.

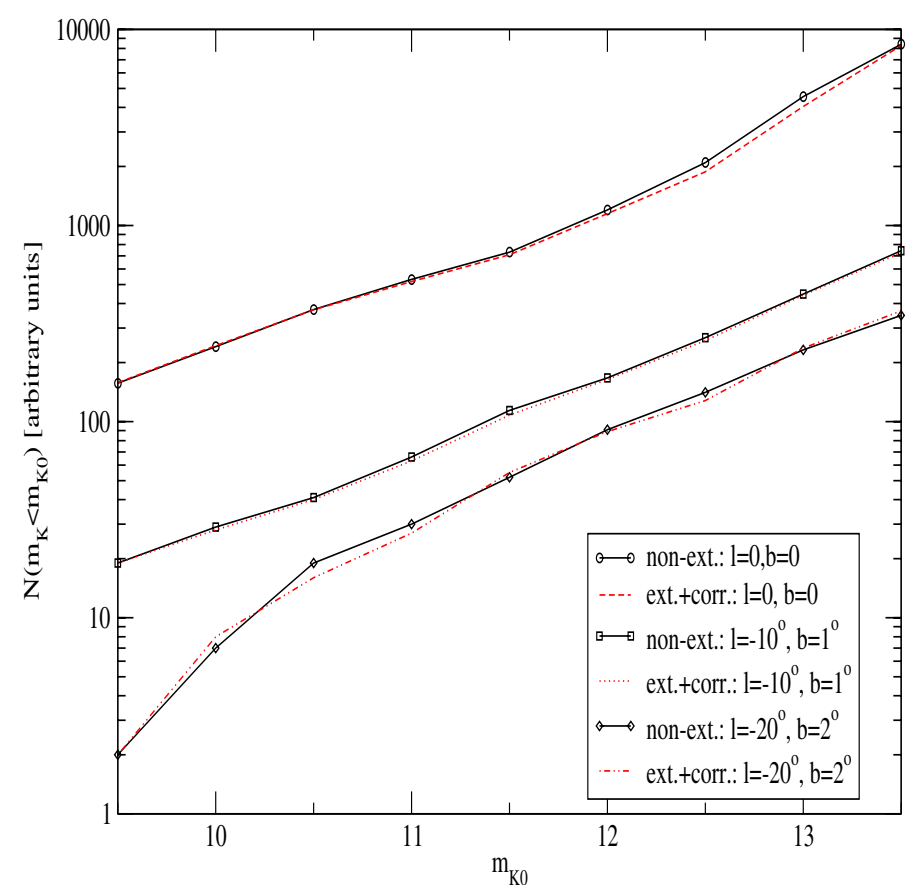

Fig. A.3. Calculations of the counts (arbitrary normalization) for the model in different regions without extinction, and with extinction+correction of the extinction. 